\section{ORIGINAL RESEARCH}

T. Hattori

K. Ito

S. Aoki

T. Yuasa

R. Sato

M. Ishikawa

H. Sawaura

M. Hori

H. Mizusawa

\title{
White Matter Alteration in Idiopathic Normal Pressure Hydrocephalus: Tract-Based Spatial Statistics Study
}

\begin{abstract}
BACKGROUND AND PURPOSE: White matter alteration in iNPH has not been well-investigated. TBSS is a voxelwise statistical analysis developed for DTI data. We aimed to elucidate the cerebral white matter alteration in patients with $\mathrm{NPH}$ by using DTI and to test the accuracy of TBSS analysis.
\end{abstract}

MATERIALS AND METHODS: DTI data were obtained from 20 patients with iNPH and 20 age- and sex-matched controls. The FA values were evaluated by using TBSS, region-of-interest and tract-specific analysis of the CST. The accuracy of TBSS analysis was tested by using "back-projection" of TBSS results and by comparing the TBSS analysis results with those of region-of-interest and tract-specific analysis.

RESULTS: Back-projection of the TBSS results showed accurate registration of the whole brain, with the exception of parts of the thalamus, fornix, and white matter around the posterior body of the lateral ventricle. The TBSS analysis results were consistent with those of the region-of-interest analysis and tract-specific analysis. In patients with iNPH compared with control subjects, the FA values were significantly decreased in parts of the corpus callosum, periventricular white matter, and juxtacortical white matter in the frontal and parietal lobes. In contrast, FA values were significantly increased in the internal capsule, extending to the white matter in the centrum semiovale.

CONCLUSIONS: Our results suggest that patients with iNPH have various patterns of white matter damage and that TBSS analysis is a promising tool for performing accurate voxelwise statistical analysis of the iNPH brain, with the exception of misregistered areas.

ABBREVIATIONS: CST $=$ corticospinal tract; $F A=$ fractional anisotropy; $\mathrm{iNPH}=$ idiopathic normal pressure hydrocephalus; $\mathrm{MNI}=$ Montreal Neurological Institute; MMSE = Mini-Mental State Examination; SLF = superior longitudinal fasciculus; TBSS = tract-based spatial statistics; TFCE = threshold-free cluster-enhancement

$\mathbf{i}_{\mathrm{i} x}^{\mathrm{N}}$ $\mathrm{NPH}$ is a clinical entity of unknown cause and is characterized by the triad of gait disturbance, urinary incontinence, and cognitive decline. ${ }^{1}$ Neuropathologic studies of chronic hydrocephalus have suggested the presence of white matter damage, ${ }^{2-4}$ presumably resulting from mechanical pressure due to ventricular enlargement ${ }^{5}$ and metabolic derangement. ${ }^{6}$

Recently, DTI has been used to quantitatively evaluate the white matter alteration in various neurodegenerative diseases in vivo. ${ }^{7,8}$ Diffusion anisotropy is most commonly quantified by using FA values. ${ }^{9}$ There are various methods of analyzing DTI data, such as region-of-interest analysis, tract-specific analysis, and voxelwise statistical analysis. In region-of-interest analysis, values are measured in any structure of interest. However, region-of-interest analysis may be biased by subjectivity, because it is sometimes difficult to objectively and reproducibly place regions of interest. In contrast, tract-specific

Received March 31, 2011; accepted after revision April 25.

From the Department of Neurology and Neurological Sciences (T.H., H.M.), Graduate School, Tokyo Medical and Dental University, Tokyo, Japan; Department of Radiology (T.H., K.I., S.A., R.S., M.I., M.H.), Juntendo University, Tokyo, Japan; Departments of Neurology (T.H., T.Y.) and Neurosurgery (H.S.), Kamagaya General Hospital, Chiba, Japan; Department of Neurology (T.H.), Kanto Central Hospital, Tokyo, Japan; Department of Radiological Sciences (R.S.), Graduate School of Human Health Sciences, Tokyo Metropolitan University, Tokyo, Japan; and Image Analytical Group (M.I.), Clinical Research Department, Micron Inc, Tokyo, Japan.

Please address correspondence to Takaaki Hattori, MD, Tokyo Medical and Dental University, 1-5-45 Yushima, Bunkyo-ku, Tokyo, 113-8519, Japan; e-mail address: takaakihattori@umin.ac.jp

Indicates open access to non-subscribers at www.ajnr.org

http://dx.doi.org/10.3174/ajnr.A2706 analysis uses tractography as an anatomic landmark, and values are measured along the tractography, which is drawn by defining seed and target regions of interest. Although regionof-interest analysis and tract-specific analysis are simple methods that can be used directly to derive values for structures that are anatomically the same among individuals, then to compare these values between groups, these methods have limitations because they are sensitive only to changes in those few parts of the brain that they can accurately measure. In contrast, voxelwise statistical analysis requires neither prespecification nor prelocalization of regions of interest, and it can be used automatically to perform a statistical analysis of the whole brain by voxelwise comparisons between 2 groups. TBSS is a recently developed voxelwise statistical analysis that has a specific registration algorithm for FA maps to minimize misregistration. ${ }^{10}$

Whole-brain analysis by using voxelwise statistical analysis is likely to be useful for exploring the white matter changes that occur in patients with iNPH because the brains of these patients are likely to be diffusely affected by ventricular enlargement and/or metabolic derangement. Although there is no established method for achieving accurate registration in these patients, the use of the registration algorithm of TBSS analysis specifically developed for DTI data may be the optimum solution. In TBSS analysis, there is a function called "back-projection," which reverses each voxel on the mean FA skeleton back to its original place in native space. ${ }^{11}$ This function may be beneficial, especially for the cohort including patients with iNPH, to confirm the accuracy of registration by 


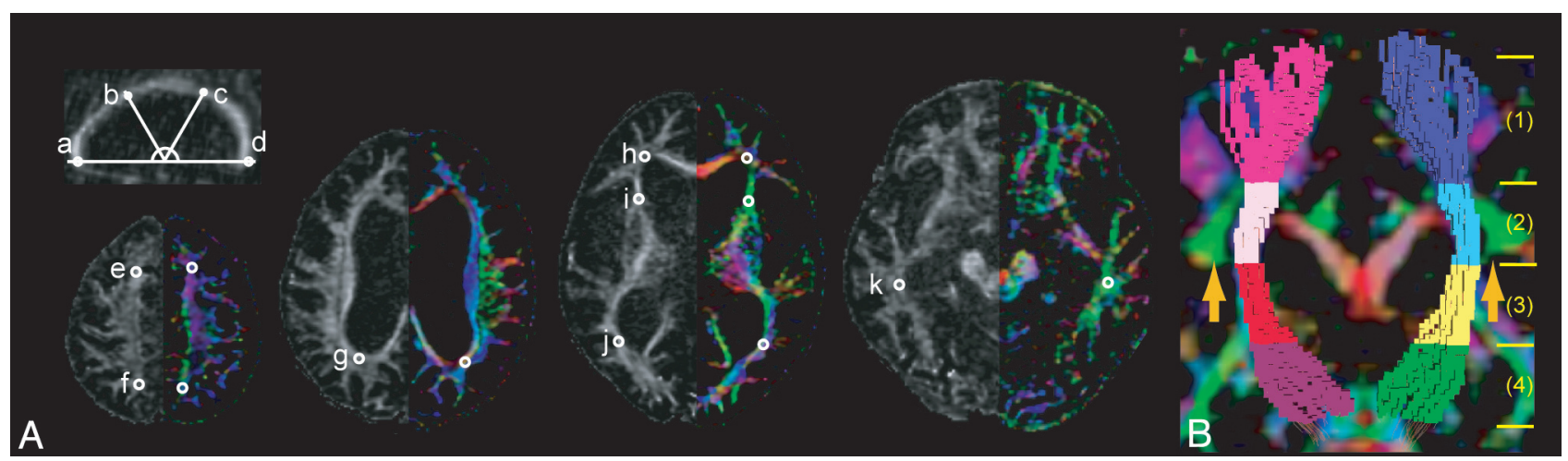

Fig 1. Region-of-interest analysis and tract-specific analysis of the CST. A, Regions of interest were plotted on the 4 points of the corpus callosum (a), the genu, anterior third of the body (b), the posterior third of the body (c), and splenium (d) on the sagittal section (FA map), the juxtacortical white matter in the frontal (e) and parietal cortices (f), the periventricular white matter around the posterior part $(\mathrm{g})$ or anterior horn $(\mathrm{h})$ of the lateral ventricle, the anterior limbs of the internal capsule (i), and the periventricular white matter around the posterior horn of the lateral ventricle (j) white matter in the temporal lobe (k) on the axial section (left, FA map; right, color-coded FA map). $B$, The voxelized diffusion tensor tractography of the CST is separated into 4 areas, (1), (2), (3), and, (4) shown with a color-coded FA map (coronal section). In the color-coded FA maps, the orientation of the axial eigenvector was color-coded per voxel according to the red-green-blue convention: red indicating a predominant left-right orientation within each voxel, green indicating an anteroposterior orientation, and blue indicating a superior-inferior orientation. The SLF (green area) is indicated by arrows.

showing the direct relationship between the TBSS results for standard space and those of the native image.

We aimed to explore the white matter alteration in patients with iNPH by using various diffusion tensor metrics with TBSS analysis. We also aimed to test the accuracy of TBSS analysis by back-projection of the TBSS results and by comparing the results with those of region-of-interest analysis and tract-specific analysis.

\section{Materials and Methods}

\section{Subject Selection}

Subjects were prospectively recruited from among patients attending Kamagaya General Hospital in Chiba, Japan. All subjects were medically interviewed and were given general and neurologic examinations, including the MMSE, by neurologists (T.H. and T.Y.). For all patients, information on memory loss or other subjective cognitive deficits was gathered by means of caregiver-based interviews. iNPH was diagnosed according to the clinical diagnostic criteria of probable iNPH. ${ }^{1}$ Healthy control subjects were selected on the basis of a normal result in the MMSE and normal findings on neurologic examinations. Subjects with a history of neurologic disease (eg, stroke or epilepsy) or with underlying diseases that potentially affected the brain (eg, uncontrolled hypertension or chronic kidney disease) were excluded.

This study was approved by the institutional review board of our hospital, and written informed consent was obtained from all subjects.

\section{Staging of Leukoaraiosis}

The extent of periventricular leukoaraiosis was rated in all subjects in accordance with the classification of Fazekas et al. ${ }^{12}$

\section{DTI Data Acquisition}

Diffusion tensor images were obtained with a $1.5 \mathrm{~T}$ clinical scanner (Signa Excite; GE Healthcare, Milwaukee, Wisconsin) with a diffusion-weighted single-shot echo-planar imaging ( $\mathrm{TR}=14,000 \mathrm{~ms}$; $\mathrm{TE}=103.5 \mathrm{~ms} ; b=1000 \mathrm{~s} / \mathrm{mm}^{2} ; 13$ different axes of motion-probing gradients; FOV $=280 \mathrm{~mm}$; matrix $=128 \times 128$ [interpolated image matrix size, $256 \times 256]$; section spacing $=3 \mathrm{~mm}$ without gap; aver- aging $=2$; number of sections $=50$; total acquisition time $=6$ minutes 32 seconds).

\section{Region-of-Interest Analysis}

DTI data were analyzed by using dTV II and Volume-One 1.72, software developed by Masutani et al (http://www.ut-radiology.umin.jp/ people/masutani/dTV.htm) and running on Windows XP Professional (Microsoft, Redmond, Washington). We chose a region of interest anatomically by using an FA map and a color-coded FA map. Regions of interest were placed anatomically in a number of areas (Fig $1 A$ ). The region of interest was set as a sphere $2 \mathrm{~mm}$ in diameter in the anterior or posterior third of the body of the corpus callosum. A sphere $3 \mathrm{~mm}$ in diameter was used in other areas. The FA value was measured in these regions of interest.

\section{Tract-Specific Analysis of the CST}

We evaluated the CST by using tract-specific analysis, because the tractography of the CST was stably drawn and could be divided into sections. ${ }^{13,14}$ Data were analyzed by using the above-mentioned dTV II and Volume-One 1.72. The threshold of line-tracking was set at FA $>0.18$. Diffusion tensor tractography of the CST was generated by using a 2-region-of-interest method, with the seed region of interest on the motor cortex and the target region of interest on the cerebral peduncle. ${ }^{13}$ Voxelization along the CST was performed between the motor cortex and the cerebral peduncle by using dTV (Fig $1 B$ ). The CST crosses the SLF at the level of the corona radiata. ${ }^{15}$ As an anatomic landmark for the CST, we used the uppermost part of the SLF, according to a previous study. ${ }^{13}$ We separated the CST into 4 areas (1, 2,3 , and 4 ) for identification. Area 1 was defined as the area of the CST above the uppermost part of the SLF. Areas 2, 3, and 4 were defined as equal one-third divisions of the CST from the uppermost part of the SLF to the cerebral peduncle. The FA value was measured in the segmented areas of the CST by using tract-specific analysis.

\section{TBSS Analysis}

Voxelwise statistical analysis of the DTI data was performed by using TBSS $^{10}$ implemented in the Functional MR Imaging of the Brain Software Library 4.1.6 (University of Oxford, Oxford, UK). ${ }^{16}$ FA images were created after correcting the DTI images for motion and eddy 


\begin{tabular}{|c|c|c|c|}
\hline & iNPH $(n=20)$ & Control $(n=20)$ & $P$ Value $^{\mathrm{a}}$ \\
\hline \multicolumn{4}{|l|}{ General characteristics } \\
\hline Sex (male/female) (No.) & $8 / 12$ & $8 / 12$ & 1.000 \\
\hline Age (mean) (yr) & $77.0 \pm 5.1$ & $73.4 \pm 6.3$ & .054 \\
\hline \multicolumn{4}{|l|}{ Disease characteristics } \\
\hline Gait disturbance $>2$ years (№.) & $100 \%(20)$ & $0 \%$ & \\
\hline Dementia >1 year (No.) & $90 \%(18)$ & $0 \%$ & \\
\hline Urinary incontinence (No.) & $50 \%(10)$ & $0 \%$ & \\
\hline \multicolumn{4}{|l|}{ Psychometric tests } \\
\hline MMSE (mean) & $21.8 \pm 3.8$ & $28.7 \pm 1.0$ & $<.001$ \\
\hline \multicolumn{4}{|l|}{ Complicating diseases } \\
\hline Hypertension (No.) & $30 \%(6)$ & $40 \%(8)$ & .736 \\
\hline Diabetes mellitus (№.) & $15 \%(3)$ & $5 \%(1)$ & .292 \\
\hline Dyslipidemia (№.) & $25 \%(5)$ & $10 \%(2)$ & .212 \\
\hline Evans index (mean) & $0.36 \pm 0.05$ & $0.28 \pm 0.03$ & $<.001$ \\
\hline Extent of leukoaraiosis (Fazekas classification) & $2.1 \pm 0.9$ & $0.8 \pm 0.8$ & $<.001$ \\
\hline
\end{tabular}

${ }^{a}$ Unpaired Student $t$ test.

current effect by using 12-parameter affine registration to the nondiffusion volumes $(b=0)$. For the registration algorithm, TBSS analysis has an option that explores the "study-specific representative image" to minimize the amount of warping required for all other subjects to align to it. ${ }^{10}$ For this purpose, every FA image was aligned to every other one to identify the most representative one, and this image was used as the target image. This target image was then affine-aligned into MNI 152 standard space, and every image was transformed into $1 \times 1 \times 1 \mathrm{~mm}$ MNI 152 space by combining the nonlinear transform to the target FA image with the affine transform from the target native space to MNI 152 space. The mean FA image was created and thinned to create the mean FA skeleton, which represented the centers of all tracts common to the groups. This skeleton was thresholded at FA $>$ 0.25 . Each subject's aligned FA map was then projected onto the skeleton by assigning each point on the skeleton the maximum FA in a plane perpendicular to the local skeleton structure. The resulting skeletons were fed into voxelwise statistics. The number of permutations was set to 5000. By using the TBSS results for the FA maps, we also analyzed maps of axial eigenvalues, radial eigenvalues, and ADC values by TBSS analysis.

The TBSS results of FA map were back-projected to each native FA map by using the "tbss_deproject" command of the FSL. Back-projection was performed by using the following 2 processes: First, all voxels were projected back from their positions on the mean FA skeleton to the nearby position at the center of the nearest tract in the subject's FA image in standard space; second, these points were brought back into the subject's native space by inverting the nonlinear registration that was applied in the TBSS analysis (see details at http://www.fmrib.ox.ac.uk/fsl/tbss/ index.html). The back-projected TBSS results were superimposed on the native FA map of each subject. A neurologist (T.H.) and a neuroradiologist (S.A.) then visually assessed whether the back-projected TBSS results were located at the centers of tracts that were anatomically the same among subjects. When the back-projected TBSS results were located in the lateral ventricle or in structures that differed among subjects, we considered that the areas were misregistered. The 2 raters (T.H. and S.A.) identified misregistered areas in the mean FA skeleton on the standard space by consensus.

\section{Statistical Analysis}

Statistical analysis between groups was performed by using an unpaired $t$ test for continuous variables and a $\chi^{2}$ test for categoric data. A Bonferroni correction was used for multiple comparisons for region- of-interest analysis and tract-specific analysis. Analyses were performed with Statistical Package for Social Sciences, Version 11 (SPSS, Chicago, Illinois).

Diffusion tensor metrics were compared between groups by using TBSS analysis. The significance threshold for between-group differences was set at $P<.05$; this was corrected for multiple comparisons across voxels by using the TFCE option.

\section{Results}

\section{Demographic and Imaging Data}

We enrolled 20 patients with iNPH and 20 age- and sexmatched control subjects. All patients with iNPH showed gait disturbance and a varying degree of cognitive dysfunction and incontinence. All these patients had communicating hydrocephalus (Evans index $>0.3$ ) and narrowed CSF spaces with high convexity on MR imaging. A spinal tap test was performed, and symptomatic improvement was confirmed in all patients. A shunt operation was performed, and symptomatic improvement was confirmed in 12 patients with iNPH. A shunt operation was not performed in 8 patients with iNPH. The demographic and imaging data on patients with iNPH and control subjects are listed in Table 1. There were no significant differences between the groups in age, sex, or vascular risk factors. Patients with iNPH had significantly more extensive leukoaraiosis than control subjects $(P<.001)$.

\section{Results of Region-of-Interest Analysis and Tract-Specific Analysis of the CST}

The results of the region-of-interest analysis and tract-specific analysis are shown in Table 2. In the region-of-interest analysis, FA values were significantly lower $(P<.001)$ in patients with iNPH than in control subjects in the posterior third of the body (c) and the splenium (d) of the corpus callosum; bilaterally in the juxtacortical white matter of the frontal (e) and parietal (f) lobes; in the periventricular white matter around the posterior part $(\mathrm{g})$, the anterior horn $(\mathrm{h})$ or the posterior horn $(\mathrm{j})$ of the lateral ventricle. In the tract-specific analysis, the FA values in patients with iNPH were significantly lower $(P<.001)$ than those in the controls bilaterally in area 1 of the 
Table 2: ROI analysis and tract-specific analysis of FA values in patients with iNPH and control subjects ${ }^{\mathrm{a}}$

\begin{tabular}{llc}
\hline & iNPH $(n=20)$ & Controls $(n=20)$ \\
\hline ROI analysis & & \\
Corpus callosum: & $0.71 \pm 0.07$ & $0.78 \pm 0.06$ \\
a) Genu & $0.58 \pm 0.07$ & $0.64 \pm 0.06$ \\
b) Anterior third of body & $0.55 \pm 0.09^{\mathrm{b}}$ & $0.69 \pm 0.06$ \\
c) Posterior third of body & $0.68 \pm 0.11^{\mathrm{b}}$ & $0.78 \pm 0.05$ \\
d) Splenium & $0.50 \pm 0.03^{\mathrm{b}} / 0.50 \pm 0.04^{\mathrm{b}}$ & $0.61 \pm 0.04 / 0.62 \pm 0.04$ \\
e) Juxtacortical WM in frontal lobe & $0.44 \pm 0.04^{\mathrm{b}} / 0.44 \pm 0.05^{\mathrm{b}}$ & $0.58 \pm 0.04 / 0.56 \pm 0.06$ \\
f) Juxtacortical WM in parietal lobe & $0.27 \pm 0.07^{\mathrm{b}} / 0.27 \pm 0.06^{\mathrm{b}}$ & $0.39 \pm 0.07 / 0.34 \pm 0.05$ \\
g) WM around posterior horn of lateral ventricle & $0.25 \pm 0.07^{\mathrm{b}} / 0.24 \pm 0.07^{\mathrm{b}}$ & $0.36 \pm 0.06 / 0.32 \pm 0.06$ \\
h) WM around anterior horn of lateral ventricle & $0.49 \pm 0.05 / 0.49 \pm 0.03$ & $0.48 \pm 0.04 / 0.49 \pm 0.05$ \\
i) Anterior limbs of internal capsule & $0.48 \pm 0.03^{\mathrm{b}} / 0.48 \pm 0.03^{\mathrm{b}}$ & $0.60 \pm 0.05 / 0.61 \pm 0.04$ \\
j) WM around posterior horn of lateral ventricle & $0.51 \pm 0.04 / 0.51 \pm 0.03$ & $0.52 \pm 0.04 / 0.52 \pm 0.03$ \\
k) WM in temporal lobe & & \\
Tract-specific analysis & $0.41 \pm 0.04^{\mathrm{b}} / 0.41 \pm 0.03^{\mathrm{b}}$ & $0.45 \pm 0.03 / 0.46 \pm 0.03$ \\
Area 1 & $0.53 \pm 0.06^{\mathrm{b}} / 0.55 \pm 0.05^{\mathrm{b}}$ & $0.43 \pm 0.04 / 0.44 \pm 0.05$ \\
Area 2 & $0.69 \pm 0.04^{\mathrm{b}} / 0.71 \pm 0.04^{\mathrm{b}}$ & $0.62 \pm 0.05 / 0.65 \pm 0.05$ \\
Area 3 & $0.60 \pm 0.05 / 0.62 \pm 0.05$ & $0.58 \pm 0.05 / 0.61 \pm 0.04$ \\
Area 4 & &
\end{tabular}

Note:-ROI indicates region of interest; WM, white matter.

a Values are presented as right/left.

b $p<.001$, unpaired Student $t$ test.

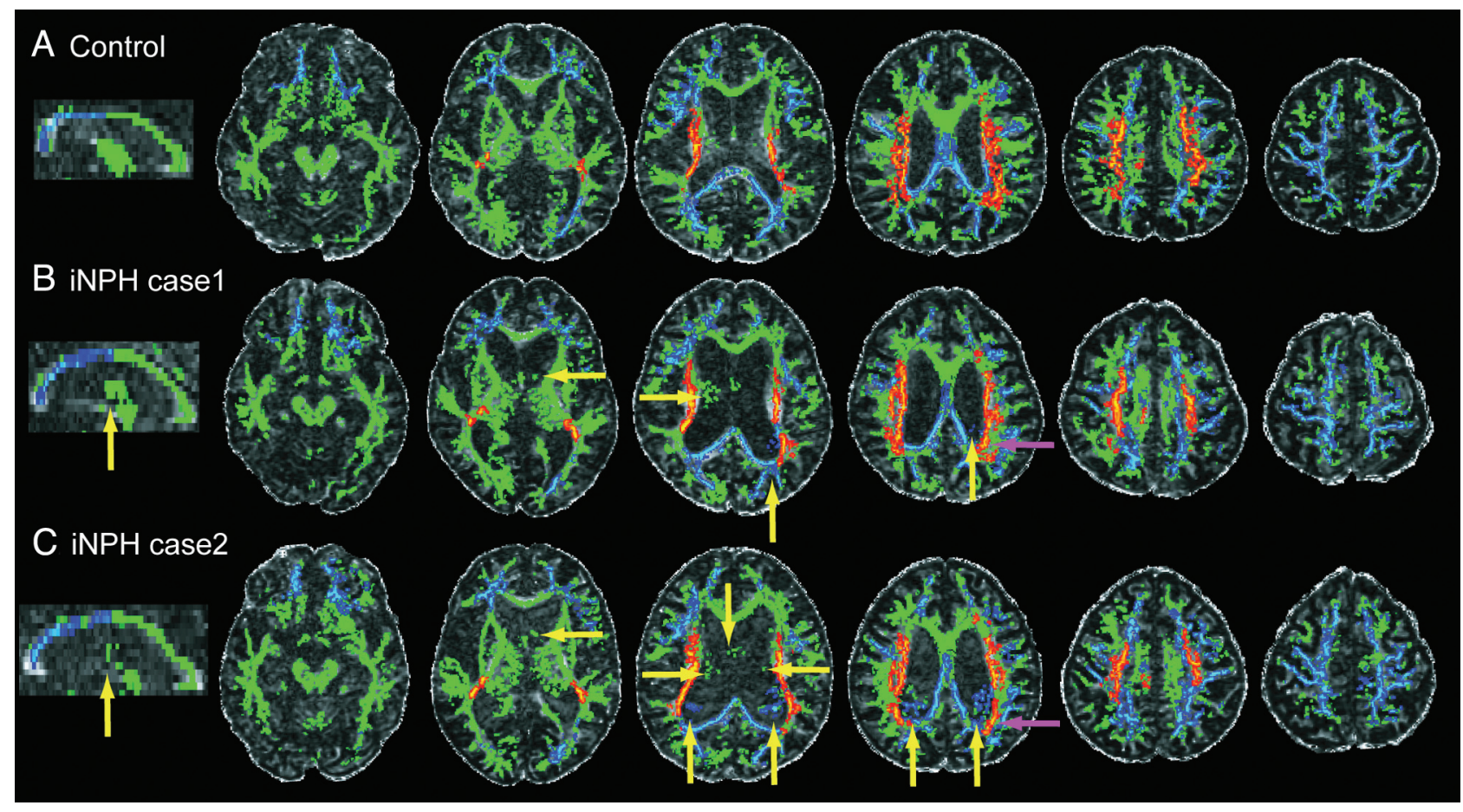

Fig 2. Examples of back-projected TBSS results of FA map native images. The back-projected image of TBSS results to native images of control subjects ( $A$ ) or patients with iNPH ( $B$ and C). The yellow or purple arrows indicate the areas where back-projected TBSS results are located in the lateral ventricle or on the different structures among subjects, respectively.

CST and significantly higher $(P<.001)$ bilaterally in areas 2 and 3 of the CST.

\section{Results of Back-Projection of TBSS}

Examples of back-projected TBSS results of FA map are given in Fig 2. In all control subjects, back-projected TBSS results were located on the appropriate structures in native space (Fig 2A). In patients with iNPH, back-projected TBSS results were located on the appropriate structures in native space, except in the following areas: around the thalamus (20 patients with iNPH) or fornix (20 patients with iNPH) in the lateral ventricle or in the posterior body of the lateral ventricle (13 patients with iNPH) (yellow arrows in Fig $2 B,-C$ ). In addition, some of the back-projected TBSS results were located on different structures in different subjects. In the white matter around the posterior body of the lateral ventricle, some of the TBSS back-projections showing significantly greater FA values in patients with iNPH than in control subjects (as shown by red to yellow) were located on the lateral juxtaventricular tracts in all control subjects, whereas, in 13 patients with iNPH, they were located on the medial juxtaventricular tracts (purple arrows in Fig $2 B,-C)$. 


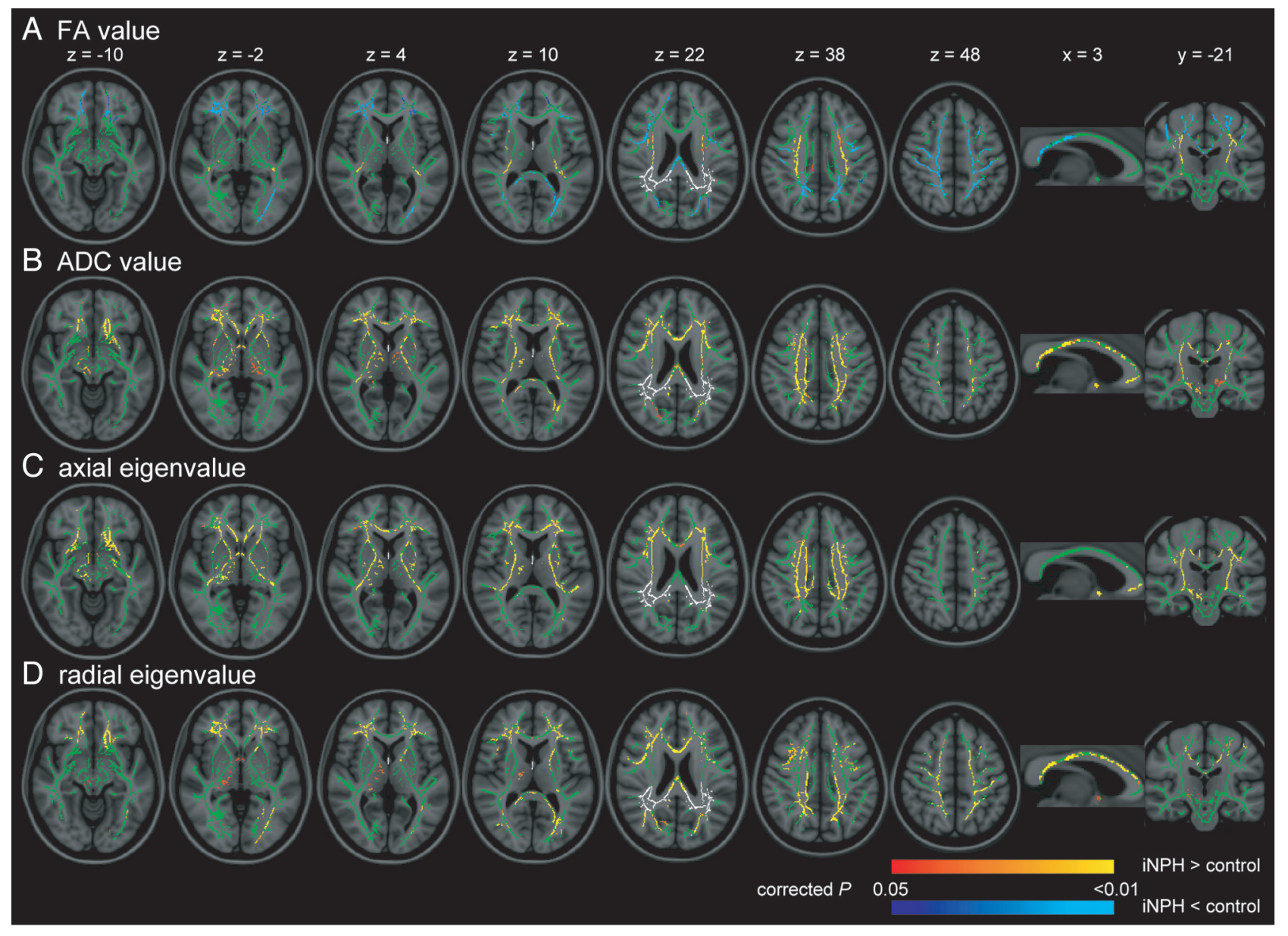

Fig 3. TBSS results. Areas with significantly decreased or increased values are shown by colors ranging from blue to light blue or red to yellow, respectively, in patients with iNPH compared with control subjects $(P<.05$, corrected for multiple comparisons by using TFCE). The areas without alteration are shown with green. The misregistrated areas are shown in white.

\section{Results of TBSS Analysis}

The TBSS results are shown in Fig 3. Areas misregistered in the mean FA skeleton in standard space were identified by using back-projection of TBSS results, and they are shown in white.

FA values in the juxtacortical white matter in the frontal and parietal lobes, the periventricular white matter, and the posterior parts of the body or splenium of the corpus callosum were significantly lower in patients with iNPH than in control subjects (corrected $P<.05$ ). In contrast, in patients with $\mathrm{iNPH}$, the FA values in the internal capsule extending to the white matter in the centrum semiovale, including the CST, were significantly higher than those in the controls (corrected $P<.05)$.

ADC values in the periventricular white matter, corpus callosum, parts of the internal capsule, and parts of the uncinate fasciculus were significantly greater in patients with iNPH than in control subjects (corrected $P<.05$ ).

Axial eigenvalues in the internal capsule extending to the white matter in the centrum semiovale as well as in the genu of the corpus callosum, parts of the cingulum, and the periventricular white matter around the anterior horn of the lateral ventricle were significantly higher in patients with iNPH than in control subjects (corrected $P<.05$ ).

Radial eigenvalues in the juxtacortical white matter in the frontal and parietal lobes and in the periventricular white matter and most parts of the corpus callosum were significantly higher in patients with iNPH than in control subjects (corrected $P<.05$ ).

The results of TBSS analysis were consistent with those of region-of-interest analysis or tract-specific analysis of the CST in areas that were anatomically the same.

\section{Discussion}

We first evaluated the various diffusion tensor metrics in the whole brains of patients with iNPH by using TBSS analysis. We then evaluated the accuracy of TBSS analysis by using back-projection of the TBSS results and by comparing the results with those of region-of-interest analysis and tract-specific analysis of the CST.

Back-projection of TBSS results demonstrated accurate registration of the whole brain, with the exception of some misregistered areas. In a few areas, the TBSS results were backprojected onto inappropriate structures (ie, the areas around the thalamus or fornix in the lateral ventricle or in the posterior body of the lateral ventricle). We considered that structures close to these areas in native space (such as parts of the thalamus, fornix, and white matter around the posterior body of the lateral ventricle) were the parts misregistered in standard space. In patients with iNPH, the thalamus and white matter around the posterior body of the lateral ventricle are strongly deviated because of enlargement of the lateral ventricle. These strong deformations might, therefore, have resulted 
in misregistration of these areas in the TBSS analysis. In addition, the fornix is attached to the posterior part of the body and splenium of the corpus callosum, ${ }^{17}$ and in patients with iNPH, it is presumably stretched and displaced by the elevated and stretched corpus callosum. ${ }^{18}$ Thus, the altered morphology of the fornix in patients with iNPH might have resulted in misregistration in the TBSS analysis. In addition, parts of the back-projected TBSS results were located on different structures in the white matter around the posterior body of the lateral ventricle in different subjects. We considered that these areas were also misregistered in the TBSS analysis.

For cross-validation purposes, we compared the results of the TBSS analysis, region of interest analysis, and tract-specific analysis of the CST for FA values. The results of the TBSS analysis were consistent with those of the region-of-interest analysis and the tract-specific analysis of the CST. In addition, other diffusion tensor metrics, such as ADC values and axial and radial eigenvalues, were measured in the same regions of interest or by using tract-specific analysis in the same areas of the CST (data not shown), and the results were also consistent with those of the TBSS analysis. These results support the validity of TBSS analysis for various diffusion tensor metrics.

In our entire cohort, there were various brain morphologies. We, therefore, considered that the registration option of TBSS analysis, which explores a study-specific representative image as a target image for nonlinear registration, was appropriate for minimizing misregistration. ${ }^{10}$ In addition, we enrolled the same number of patients with iNPH $(n=20)$ and control subjects $(n=20)$ to exclude the potential biases resulting from a difference in subject numbers that might affect the selection of study-specific representative targets and the TBSS results. The results overall indicate that the TBSS analysis was appropriately performed in this study, except in the misregistered areas.

FA values in parts of the corpus callosum and in the deep white matter and juxtacortical white matter were significantly lower in patients with iNPH than in control subjects. The multifaceted pathologies seen in patients with iNPH may underlie these decreased FA values. First, the mechanical pressure resulting from ventricular enlargement stretches and compresses the periventricular white matter ${ }^{4}$ and the corpus callosum, ${ }^{18,19}$ causing axonal loss. ${ }^{3}$ Second, there may be interstitial edema, predominantly in the periventricular white matter, ${ }^{20}$ owing to suffusion of CSF. In addition to this decrease in FA values, $\mathrm{ADC}$ values and radial eigenvalues in the periventricular white matter were significantly greater in patients with iNPH than in the controls, supporting the presence of free water and/or interstitial edema in the white matter of patients with iNPH. Increased ADC values in the periventricular white matter were also shown in a previous region-of-interest analysis of hydrocephalus. ${ }^{21}$ Third, the diffuse metabolic derangements in iNPH that are caused by reduced CSF turnover, with accumulation of neurotoxic substances, such as $\beta$-amyloid, $\tau$ protein, and pro-inflammatory cytokines, may also cause diffuse cerebral white matter damage. ${ }^{6}$ Cortical biopsy samples taken at the time of shunt placement in patients with iNPH show the presence of Alzheimer disease - type pathologies. $^{22,23}$ These findings may underlie the decreased FA values that we found in the structures beyond the periventricular white matter. Our patients with iNPH had signifi- cantly more extensive periventricular leukoaraiosis than the control subjects. The presence of leukoaraiosis may also be, at least in part, related to the altered diffusion tensor metrics in the periventricular white matter in patients with iNPH.

The TBSS analysis showed that FA values and axial eigenvalues in the internal capsule extending to the white matter in the centrum semiovale, including the CST, were significantly greater in patients with iNPH than in controls, whereas the radial eigenvalues were not significantly altered. In general, FA values are positively correlated with axial eigenvalues and negatively correlated with radial eigenvalues. ${ }^{24}$ Therefore, the increased FA values in patients with iNPH were due mainly to the increase in axial eigenvalues. To explain the increased axial eigenvalues, we speculate as follows: The brain parenchyma is stretched and compressed by mechanical pressure from ventricular enlargement in patients with iNPH, resulting in stretching of the neural fibers. In the altered microstructure, neural fibers microscopically show reduced winding and a tendency to align in the direction of stretch. Thus, water diffusivity parallel to the axon is enhanced, leading to an increase in axial eigenvalues. In addition, decreased integrity of crossing fibers may contribute to the increase in FA values. In general, FA values decrease at points where fibers $\operatorname{cross}^{9}$ but FA values increase when the crossing fibers are lost and only the fibers of interest are left.

The CST crosses the interhemispheric fibers as well as the SLF at the level of the corona radiata. ${ }^{15}$ Interhemispheric fibers pass through the corpus callosum. In iNPH, the mechanical pressure due to ventricular enlargement stretches and compresses the corpus callosum, ${ }^{18,19}$ and the result of this pressure may cause axonal loss and wallerian degeneration of the interhemispheric fibers. In fact, our TBSS results showed that various diffusion tensor metrics were significantly altered in the corpus callosum in patients with iNPH compared with control subjects, suggesting that the interhemispheric fibers passing through the corpus callosum were damaged. In addition, the FA values were significantly decreased in the frontal and parietal juxtacortical white matter, which may include parts of the SLF projecting to these broad areas. Thus, decreased integrity of crossing fibers may also contribute to the increase in FA values in the internal capsule extending to the white matter in the centrum semiovale.

The origin of the gait disturbance in patients with iNPH is not entirely understood. The early hypothesis was compression and/or deformation of the CST due to enlargement of the ventricles. ${ }^{1}$ Our study showed that the diffusion metrics of the CST were altered in patients with iNPH, suggesting microstructural alteration of the CST. However, the involvement of the CST in gait disturbance in patients with iNPH has been controversial in previous studies. ${ }^{18,25,26}$ In contrast, a recent hypothesis for gait disturbance in patients with iNPH is frontal lobe dysfunction ${ }^{27,28}$ and/or malfunction of the cortex-basal ganglia-thalamus-cortex circuit, including the supplementary motor cortex. ${ }^{29}$ On the other hand, the origin of dementia in patients with iNPH is also unknown. Patients with iNPH can have psychomotor slowing, impaired attention, executive dysfunction, and visuospatial dysfunction. ${ }^{30}$ These features resemble those seen in frontal disorders ${ }^{31,32}$ and are sometimes characterized as frontal and subcortical deficits. ${ }^{33}$ To date, frontal lobe dysfunction ${ }^{27,30,34}$ or prefrontal lobe discon- 
nection or both ${ }^{35}$ have been proposed to be involved in the pathogenesis of dementia in patients with iNPH.

Our results show that there are broad white matter alterations, including in the frontal lobe and basal ganglia, in patients with iNPH. As discussed above, altered diffusion tensor metrics indicate the presence of abnormal white matter pathologies. These microstructural alterations of the white matter may, at least in part, underlie the gait disturbance and/or dementia via cortical-cortical/subcortical disconnection. Further studies including of the larger numbers of cases, are needed to explore the origins of clinical signs in patients with iNPH by evaluating the correlation between diffusion tensor metrics and clinical parameters.

This study had a number of limitations. First, only limited information on the pathology of iNPH is available, and pathologic substrates for the altered diffusion tensor metrics should be investigated in a future study. Second, although we carefully identified the areas misregistered in standard space by using back-projection of the TBSS results, there may have been other misregistered areas that were underestimated, especially in the brain parenchyma.

\section{Conclusions}

We demonstrated that various diffusion tensor metrics were significantly altered in the broad white matter of patients with iNPH compared with those in control subjects. The accuracy of TBSS analysis was confirmed in the whole brain, except the misregistered areas. These findings suggest that patients with iNPH have various patterns of white matter damage and that TBSS analysis is a promising tool for use in accurate voxelwise statistical analysis of the brains of patients with iNPH, except in the areas that were misregistered. The clinical relevance of the altered diffusion tensor metrics should be investigated in future studies.

\section{Acknowledgments}

We thank the "Comprehensive Brain Science Network" of the Japanese Ministry of Education, Culture, Sports, Science, and Technology for providing us with a good environment for our discussions.

Disclosures: Shigeki Aoki, RELATED: Grant. Grant-in-Aid for scientific research, Japan Society for the Promotion of Science. Hidehiro Mizusawa, UNRELATED: Board Membership: Shinkei, Igakusion Co, Clinical Neuroscience, Chugai-igaku Co; Comments: editorial fee; Grants/Grants Pending: Research grant (money to the Department of Neurology and Neurological Sciences, Graduate School, Tokyo Medical and Dental University, Tokyo, Japan); Payment for Lectures (including service on speakers bureaus): Tanabemitsubishi, Sanofi Aventis; Comments: honoraria for sponsored lectures.

\section{References}

1. Relkin N, Marmarou A, Klinge P, et al. Diagnosing idiopathic normal-pressure hydrocephalus. Neurosurgery 2005;57:S4-16, discussion ii-v

2. Akai K, Uchigasaki S, Tanaka U, et al. Normal pressure hydrocephalus: neuropathological study. Acta Pathol Jpn 1987;37:97-110

3. Ding Y, McAllister JP 2nd, Yao B, et al. Axonal damage associated with enlargement of ventricles during hydrocephalus: a silver impregnation study. Neurol Res 2001;23:581-87

4. Del Bigio MR, Wilson MJ, Enno T. Chronic hydrocephalus in rats and humans: white matter loss and behavior changes. Ann Neurol 2003;53:337-46

5. Del Bigio MR. Neuropathological changes caused by hydrocephalus. Acta Neuropathol 1993;85:573-85

6. Kondziella D, Sonnewald U, Tullberg M, et al. Brain metabolism in adult chronic hydrocephalus. J Neurochem 2008;106:1515-24

7. Hattori T, Orimo S, Aoki S, et al. Cognitive status correlates with white matter alteration in Parkinson's disease. Hum Brain Mapp 2011 Apr 14. [Epub ahead of print]

8. Sage CA, Peeters RR, Gorner A, et al. Quantitative diffusion tensor imaging in amyotrophic lateral sclerosis. Neuroimage 2007;34:486-99

9. Masutani $Y$, Aoki S, Abe O, et al. MR diffusion tensor imaging: recent advance and new techniques for diffusion tensor visualization. Eur J Radiol 2003;46: $53-66$

10. Smith SM, Jenkinson M, Johansen-Berg H, et al. Tract-based spatial statistics: voxelwise analysis of multi-subject diffusion data. Neuroimage 2006;31:1487505

11. Liu Y, Spulber G, Lehtimaki KK, et al. Diffusion tensor imaging and tractbased spatial statistics in Alzheimer's disease and mild cognitive impairment. Neurobiol Aging 2011;32:1558-71

12. Fazekas F, Chawluk JB, Alavi A, et al. MR signal abnormalities at $1.5 \mathrm{~T}$ in Alzheimer's dementia and normal aging. AJR Am J Roentgenol 1987;149: 351-56

13. Hattori T, Yuasa T, Aoki S, et al. Altered microstructure in corticospinal tract in idiopathic normal pressure hydrocephalus: Comparison with Alzheimer's disease and Parkinson's disease with dementia. AJNR Am J Neuroradiol. 2011;32:1681-87

14. Yasmin H, Aoki S, Abe O, et al. Tract-specific analysis of white matter pathways in healthy subjects: a pilot study using diffusion tensor MRI. Neuroradiology 2009;51:831-40

15. Wiegell MR, Larsson HB, Wedeen VJ. Fiber crossing in human brain depicted with diffusion tensor MR imaging. Radiology 2000;217:897-903

16. Smith SM, Jenkinson M, Woolrich MW, et al. Advances in functional and structural MR image analysis and implementation as FSL. Neuroimage 2004; 23(suppl 1):S208-19

17. Griffiths PD, Batty R, Reeves MJ, et al. Imaging the corpus callosum, septum pellucidum and fornix in children: normal anatomy and variations of normality. Neuroradiology 2009;51:337-45

18. Roricht S, Meyer BU, Woiciechowsky C, et al. Callosal and corticospinal tract function in patients with hydrocephalus: a morphometric and transcranial magnetic stimulation study. J Neurol 1998;245:280-88

19. Mataro M, Matarin M, Poca MA, et al. Functional and magnetic resonance imaging correlates of corpus callosum in normal pressure hydrocephalus before and after shunting. J Neurol Neurosurg Psychiatry 2007;78:395-9

20. Leliefeld PH, Gooskens RH, Braun KP, et al. Longitudinal diffusion-weighted imaging in infants with hydrocephalus: decrease in tissue water diffusion after cerebrospinal fluid diversion. J Neurosurg Pediatr 2009:4:56-63

21. Anik Y, Demirci A, Anik I, et al. Apparent diffusion coefficient and cerebrospinal fluid flow measurements in patients with hydrocephalus. J Comput Assist Tomogr 2008;32:392-96

22. Savolainen S, Paljarvi L, Vapalahti M. Prevalence of Alzheimer's disease in patients investigated for presumed normal pressure hydrocephalus: a clinical and neuropathological study. Acta Neurochir (Wien) 1999;141:849-53

23. Del Bigio MR, Cardoso ER, Halliday WC. Neuropathological changes in chronic adult hydrocephalus: cortical biopsies and autopsy findings. Can J Neurol Sci 1997;24:121-26

24. Le Bihan D, Mangin JF, Poupon C, et al. Diffusion tensor imaging: concepts and applications. J Magn Reson Imaging 2001;13:534-46

25. Zaaroor M, Bleich N, Chistyakov A, et al. Motor evoked potentials in the preoperative and postoperative assessment of normal pressure hydrocephalus. J Neurol Neurosurg Psychiatry 1997;62:517-21

26. Hattingen E, Jurcoane A, Melber J, et al. Diffusion tensor imaging in patients with adult chronic idiopathic hydrocephalus. Neurosurgery 2010;66:917-24

27. Miyoshi N, Kazui H, Ogino A, et al. Association between cognitive impairment and gait disturbance in patients with idiopathic normal pressure hydrocephalus. Dement Geriatr Cogn Disord 2005;20:71-76

28. Stolze H, Kuhtz-Buschbeck JP, Drucke H, et al. Comparative analysis of the gait disorder of normal pressure hydrocephalus and Parkinson's disease. J Neurol Neurosurg Psychiatry 2001;70:289-97

29. Lenfeldt N, Larsson A, Nyberg L, et al. Idiopathic normal pressure hydrocephalus: increased supplementary motor activity accounts for improvement after CSF drainage. Brain 2008;131:2904-12

30. Iddon JL, Pickard JD, Cross JJ, et al. Specific patterns of cognitive impairment in patients with idiopathic normal pressure hydrocephalus and Alzheimer's disease: a pilot study. J Neurol Neurosurg Psychiatry 1999;67:723-32

31. Robbins TW, James M, Owen AM, et al. Cognitive deficits in progressive supranuclear palsy, Parkinson's disease, and multiple system atrophy in tests sensitive to frontal lobe dysfunction. I Neurol Neurosurg Psychiatry 1994;57:79-88

32. Mega MS, Cummings JL. Frontal-subcortical circuits and neuropsychiatric disorders. J Neuropsychiatry Clin Neurosci 1994;6:358-70

33. Shprecher D, Schwalb J, Kurlan R. Normal pressure hydrocephalus: diagnosis and treatment. Curr Neurol Neurosci Rep 2008;8:371-76

34. Kito Y, Kazui H, Kubo Y, et al. Neuropsychiatric symptoms in patients with idiopathic normal pressure hydrocephalus. Behav Neurol 2009;21:165-74

35. Donnet A, Schmitt A, Dufour H, et al. Differential patterns of cognitive impairment in patients with aqueductal stenosis and normal pressure hydrocephalus. Acta Neurochir (Wien) 2004;146:1301-08, discussion 1308 